\title{
Programa saúde na escola: experiências, práticas e desafios na atenção primária à saúde
}

\author{
Health program at school: experiences and challenges in primary health care \\ Prácticas de apoyo a la matriz de salud mental desarrolladas en la atención primaria de salud
}

Recebido: 02/07/2021 | Revisado: 10/07/2021 | Aceito: 05/08/2021 | Publicado: 09/08/2021

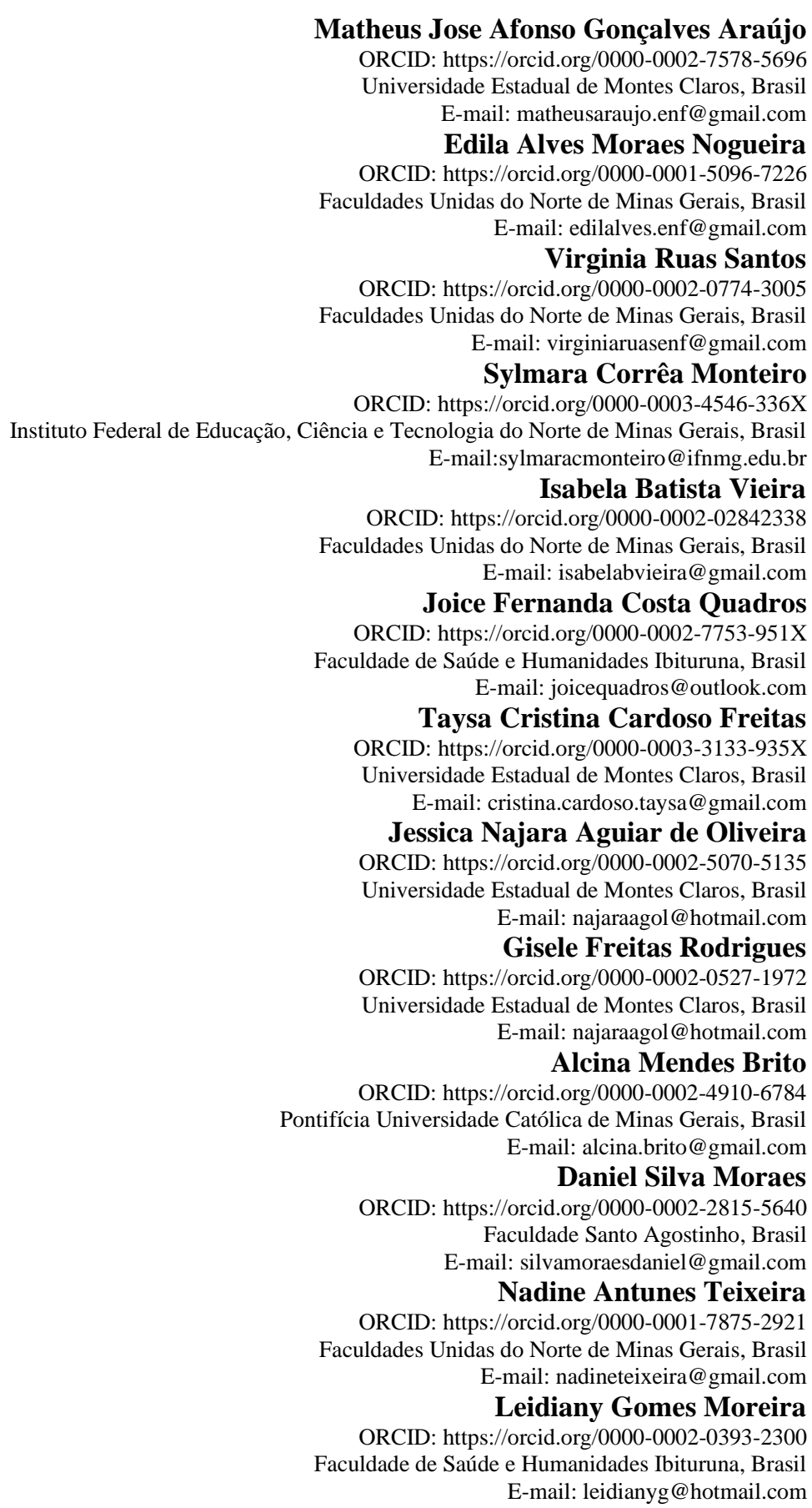

\section{Resumo}

Objetivo: Conhecer as experiências, práticas e desafios para o desenvolvimento do Programa Saúde na Escola pela Atenção Básica à Saúde. Metodologia: Conduziu-se um estudo de revisão integrativa de literatura, as bases de dados 
secundários utilizadas foram a Biblioteca Virtual em Saúde, Literatura Latino - Americana e do Caribe em Ciências da Saúde (LILACS) e a Scientific Electronic Library Online (Scielo). A busca dos artigos foi realizada por meio dos descritores Serviços de Saúde Escolar, Estratégia Saúde da Família e Atenção Básica à Saúde. Resultados: Foram encontrados 28 artigos na base de dados Biblioteca Virtual em Saúde, 63 na Scielo e 17 na LILACS, com auxílio dos critérios de inclusão e exclusão a amostra final foi composta por 17 artigos. O Programa Saúde na Escola é uma oportunidade ímpar de aproximação dos setores saúde e educação, sendo uma estratégia de promoção à saúde dos estudantes, com inúmeros benefícios. Study completion: As ações desenvolvidas nas escolas são pontuais e assistemáticas, apenas como respostas a demandas específicas, o que limita o alcance das ações do Programa Saúde na Escola.

Palavras-chave: Serviços de saúde escolar; Estratégia saúde da família; Atenção básica à saúde.

\begin{abstract}
Objective: To know the experiences, practices and challenges for the development of the School Health Program through Primary Health Care. Methodology: An integrative literature review study was conducted, the secondary databases used were the Virtual Health Library, Latin American and Caribbean Literature on Health Sciences (LILACS) and the Scientific Electronic Library Online (Scielo). The articles were searched with the descriptors School Health Services, Family Health Strategy and Primary Health Care. Results: Twenty-eight articles were found in the Virtual Health Library database, 63 in Scielo and 17 in LILACS, with the aid of inclusion and exclusion criteria, the final sample consisted of 17 articles. The School Health Program is a unique opportunity to approach the health and education sectors, being a strategy to promote students' health, with numerous benefits. Final considerations: The actions developed in schools are punctual and unsystematic, only as responses to specific demands, which limits the scope of the actions of the School Health Program.
\end{abstract}

Keywords: School health services; Family health strategy; Primary health care.

\title{
Resumen
}

Objetivo: Conocer las experiencias, prácticas y desafíos para el desarrollo del Programa de Salud Escolar a través de la Atención Primaria de Salud. Metodología: Se realizó un estudio de revisión de la literatura integrativa, las bases de datos secundarias utilizadas fueron la Biblioteca Virtual de Salud, la Literatura Latinoamericana y del Caribe sobre Ciencias de la Salud (LILACS) y la Biblioteca Electrónica Científica en Línea (Scielo). Los artículos fueron buscados con los descriptores Servicios de Salud Escolar, Estrategia de Salud Familiar y Atención Primaria de Salud. Resultados: Veintiocho artículos fueron encontrados en la base de datos de la Biblioteca Virtual en Salud, 63 en Scielo y 17 en LILACS, con la ayuda de criterios de inclusión y exclusión, la muestra final consistió en 17 artículos. El Programa de Salud Escolar es una oportunidad única para acercarse a los sectores de la salud y la educación, siendo una estrategia para promover la salud de los estudiantes, con numerosos beneficios. Finalización del estudio: Las acciones desarrolladas en las escuelas son puntuales y poco subsistemas, sólo como respuestas a demandas específicas, lo que limita el alcance de las acciones del Programa de Salud Escolar.

Palabras clave: Servicios de salud escolar; Estrategia de salud familiar; Atención primaria de salud.

\section{Introdução}

O Ministério da Saúde e Educação no ano de 2007 fundaram o Programa Saúde na Escola (PSE), com o propósito de desenvolver práticas intersetoriais entre a área da educação e saúde. O programa é direcionado a crianças, jovens e adolescentes que frequentam as instituições escolares públicas, que em muitas vezes se encontram perante vulnerabilidades sociais, interferindo no seu processo de desenvolvimento integral (Brasil, 2017).

As intervenções elaboradas nas instituições de ensino por meio do Programa Saúde na Escola são estruturadas e realizadas pelas unidades que possuem Estratégia Saúde da Família (ESF), e por profissionais que atuam na área da educação. No âmbito do Sistema Único de Saúde (SUS), a ESF é considerada como a porta de entrada da atenção primária à saúde, sendo implementada primordialmente para garantir a saúde de modo integral (Brasil, 2017).

Tendo em vista que os costumes, crenças e comportamentos no período da infância e adolescência têm grandes possibilidades de serem continuados até a fase adulta e que a maioria das crianças e dos adolescentes de nacionalidade brasileira frequentam instituições de ensino (98,6\% das crianças com faixa etária 6 a 14 anos e 85,0\% dos adolescentes de 15 a 17 anos) as instituições escolares são lugares de destaque para o desenvolvimento das práticas de prevenção de riscos e agravos, de educação em saúde e promoção à saúde (Instituto Brasileiro de Geografia e Estatística, 2016; Casemiro, Fonseca, \& Secco, 2014; Brasil, 2017). 
As instituições de ensino como ambiente de saúde coletiva atravessam por potencialidades que se confirmam tanto pela expressividade sociodemográfica associada ao período da infância e adolescência como pelo enfrentamento planejado de vulnerabilidades que atingem os alunos na esfera escolar (World health organization, 2017). O PSE tem importante função na consolidação de práticas que abrangem a educação, a saúde e as demais redes de serviços públicos no enfrentamento de vulnerabilidades que interferem na saúde de crianças e adolescentes que estão na idade escolar (Brasil, 2017).

Esse programa não refere-se apenas a uma estratégia política de intersetorialidade na área da saúde, mas sim a certeza de que os ganhos em indicadores de saúde necessitam de um vasto desenvolvimento de inclusão para a compreensão e modificação da realidade, indicando um esforço na inter-relação de diferentes saberes e atores sociais para implantação de vínculos horizontais para a superação de dificuldades em saúde (WHO, 2017).

A comunicação entre a saúde e educação deve ser essencial na prestação de uma assistência mais ampla, uma vez que a escola deve ser um espaço atuante no desenvolvimento de determinantes da saúde e empoderamento da individualidade dos estudantes (Silva, Reis \& Milhorine, 2016). O reconhecimento da necessidade de trabalho em conjunto e parcerias entre os setores saúde e educação para o alcance de melhores resultados nas políticas públicas a partir do diálogo entre as equipes, consiste em um maior entrosamento e um facilitador à realização das atividades desenvolvidas, especialmente referente a promoção da saúde e cidadania entre os escolares (Souza, Esperdião \& Medina, 2017).

A adesão ao PSE pelos setores da saúde e educação contribui para a minimização das vulnerabilidades e ameaças à saúde das crianças, adolescentes e jovens, além de que suas ações conjuntas promovem e estimulam o acompanhamento das situações de saúde, identificando suas carências a fim de resolvê-las ou diminuí-las o tanto quanto possível, proporcionando um ambiente mais saudável aos estudantes que se beneficiam do programa (Brasil, 2017).

É preciso que profissionais na área da saúde possam rever seus conceitos em relação ao que entendem sobre o Programa Saúde na Escola, uma vez que a falta de conhecimento sobre o programa por parte destes profissionais, pode ser considerado um dos motivos pelo qual ocorre pouco envolvimento dos mesmos com os profissionais da área da educação, acabando por diminuir a capacidade que estas ações possuem, limitando-as a ações pontuais, o que de certa forma limita também o direito de acessibilidade à saúde a qual as crianças, adolescentes e jovens têm direito (Brasil, Silva, Silva, \& Rodrigues, 2017).

Outro ponto importante, é que o governo pode mobilizar os recursos existentes, criando plataformas para reunir as melhores estratégias, tais como cursos e materiais educativos sobre saúde, estilo de vida e apoio psicossocial para serem direcionadas à escola (Wang, Zhang, Zhao, Zhang \& Jiang, 2020).

Um dos maiores problemas do campo da Saúde Pública é o emprego das evidências alcançadas por intermédio das variadas fontes de informações (primárias, secundárias, midiáticos e artigos científicos) a fim de aperfeiçoar, modificar e reorganizar as práticas cotidianas. Nesse sentido, as pesquisas que exibem evidências têm sido uma temática de intenso interesse dos governantes e distintos atores envolvidos na introdução de propostas e de práticas de promoção da saúde (Brasil, 2017).

Com intuito que práticas sejam capazes de ser desenvolvidas entre os colaboradores da área da saúde e educação, a parceria é primordial, com o objetivo de planejamento e execução das práticas do PSE dentro do âmbito estudantil proporcionando os conhecimentos em saúde por intermédio da promoção e prevenção da saúde (Brasil, Silva, Silva, \& Rodrigues, 2017). Frente a essa discussão, questiona-se quais as variáveis que podem dificultar ou até mesmo facilitar a implantação desse programa.

Ainda há uma escassez de pesquisas que se valem de analisar tais variáveis, razão pela qual, compreendê-los permitirá que os gestores e profissionais que fazem parte do PSE corroboram as características satisfatórias e façam a adequação das ações propostas com o objetivo de corrigir as barreiras que impossibilitam a implantação efetiva do programa (Medeiros, Pinto, Paiva, Nascimento, Rebouças, \& Silva, 2018). Assim, o objetivo do presente estudo foi conhecer as experiências, práticas e desafios para o desenvolvimento do Programa Saúde na Escola pela Atenção Básica à Saúde. 


\section{Metodologia}

Foi conduzido um estudo de revisão integrativa da literatura. Para a condução da presente revisão de literatura foram seguidas seis etapas interrelacionadas e interdependentes (Soares, 2005). A pergunta guia construída foi: Quais as experiências, práticas e desafios para o desenvolvimento do Programa Saúde na Escola pela atenção básica à saúde?

A busca e seleção e de artigos foi realizada durante o mês de abril e maio de 2021, tendo como cenário de busca as bases de dados secundários Literatura Latino - Americana e do Caribe em Ciências da Saúde (LILACS), Scientific Electronic Library Online (Scielo) e da Biblioteca Virtual em Saúde (BVS).

Para a seleção dos artigos utilizou-se um instrumento construído e validado composto pelos itens: dados de identificação do artigo (título, autores, nome do periódico, ano de publicação, volume e número), tipo de estudo, local de estudo, objetivo do estudo, eixo temático, classificação Qualis/Capes, periódico de publicação, nível de evidência, desfechos, dentre outros itens.

Utilizou-se como descritores os termos selecionados através dos Descritores em Ciências da Saúde, dessa forma, os descritores guia foram Serviços de Saúde Escolar, Estratégia Saúde da Família e Atenção Básica à Saúde. Foi utilizado o operador booleano "and" para conjugação. Na busca inicial foram identificadas 136 publicações potencialmente elegíveis para a amostragem final, sendo 28 artigos na base de dados Biblioteca Virtual em Saúde, 63 na Scielo e 17 na LILACS.

Para composição da amostra final de estudos foram considerados como critérios de inclusão: artigos completos, acesso gratuito a versão completa do artigo, texto em português, inglês ou espanhol e artigos publicados nos últimos cinco anos. Os artigos selecionados foram analisados na íntegra, inicialmente, foi realizada análise dos títulos e resumos, sendo em seguida realizada a leitura completa.

Os critérios de exclusão estabelecidos foram: teses, dissertações, livros, cursos livres, textos incompletos e que não abordassem de forma direta a temática objeto do estudo, uma vez que muitos estudos abordaram apenas ações pontuais nas escolas. Assim, após considerar-se os critérios de inclusão e exclusão foram selecionados para a amostra final da presente investigação 17 artigos.

\section{Resultados}

Apresenta-se as informações extraídas dos artigos selecionados para análise, sendo organizados de acordo com o autor e ano de publicação, título do periódico, delineamento, objetivo principal do estudo e principais resultados do estudo (Quadro 1).

Quadro 1 - Artigos selecionados para análise após aplicação dos critérios de pesquisa.

\begin{tabular}{|c|c|c|c|c|c|}
\hline Autor(es)/Ano & Título & Periódico & Delineamento & Objetivo & Principais resultados \\
\hline $\begin{array}{l}\text { Silveira, Estermann, \& } \\
\text { Félix. (2019) }\end{array}$ & $\begin{array}{l}\text { A generificação } \\
\text { da } \\
\text { intersetorialidade } \\
\text { no Programa } \\
\text { Saúde na Escola. }\end{array}$ & $\begin{array}{lr}\text { Revista } & \text { Brasileira } \\
\text { de } & \text { Estudos } \\
\text { Pedagógicos. }\end{array}$ & $\begin{array}{l}\text { Trata-se de um estudo } \\
\text { que utiliza pesquisa } \\
\text { documental e análise } \\
\text { cultural. }\end{array}$ & $\begin{array}{l}\text { Discutir como o } \\
\text { gênero atravessa e } \\
\text { dimensiona um de } \\
\text { seus princípios } \\
\text { organizadores a } \\
\text { intersetorialidade. }\end{array}$ & $\begin{array}{l}\text { No PSE, noções como } \\
\text { "somar esforços", "unir- } \\
\text { se" e "articular-se" são } \\
\text { mobilizadas para propor } \\
\text { modos de fazer educação } \\
\text { e(m) saúde. }\end{array}$ \\
\hline $\begin{array}{l}\text { Batista, Mondini, \& } \\
\text { Jaime. (2017) }\end{array}$ & $\begin{array}{lr}\text { Ações } & \text { do } \\
\text { Programa } & \text { Saúde } \\
\text { na Escola } & \text { e da } \\
\text { alimentação } & \\
\text { escolar } & \text { na } \\
\text { prevenção } & \text { do }\end{array}$ & $\begin{array}{l}\text { Epidemiologia e } \\
\text { Serviços de Saúde. }\end{array}$ & Estudo descritivo. & 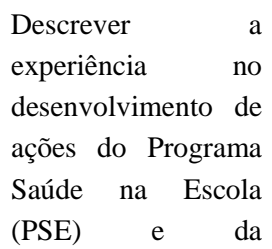 & $\begin{array}{l}\text { A avaliação das ações do } \\
\text { PSE e da alimentação } \\
\text { escolar indica a } \\
\text { necessidade de ajustes no } \\
\text { cardápio escolar. }\end{array}$ \\
\hline
\end{tabular}




\begin{tabular}{|c|c|c|c|c|c|}
\hline & 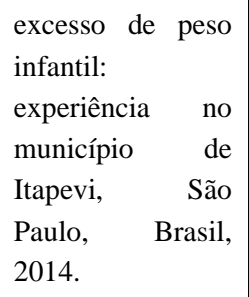 & & & $\begin{array}{lr}\text { alimentação escolar } \\
\text { relacionadas } \\
\text { prevenção à } \\
\text { excesso de peso no } \\
\text { município de Itapevi- } \\
\text { SP, Brasil. }\end{array}$ & \\
\hline $\begin{array}{l}\text { Farias, Sá, Figueiredo, } \\
\text { \& Filho (2016) }\end{array}$ & $\begin{array}{l}\text { Análise da } \\
\text { Intersetorialidade } \\
\text { no Programa } \\
\text { Saúde na Escola. }\end{array}$ & $\begin{array}{ll}\text { Revista } & \text { Brasileira } \\
\text { de } & \text { Educação } \\
\text { Médica. } & \end{array}$ & $\begin{array}{l}\text { Trata-se de uma pesquisa } \\
\text { qualitativa. }\end{array}$ & $\begin{array}{l}\text { Analisar e Conhecer } \\
\text { o processo da } \\
\text { intersetorialidade no } \\
\text { Programa Saúde na } \\
\text { Escola (PSE)em um } \\
\text { município da região } \\
\text { metropolitana de } \\
\text { Pernambuco. }\end{array}$ & $\begin{array}{l}\text { O maior dilema é operar } \\
\text { uma política } \\
\text { necessariamente } \\
\text { intersetorial, explicitada } \\
\text { num ambiente com atores } \\
\text { que possuem agendas } \\
\text { setoriais previamente } \\
\text { montadas. }\end{array}$ \\
\hline $\begin{array}{l}\text { Brambilla, Kleb, \& } \\
\text { Magroet. (2020) }\end{array}$ & \begin{tabular}{lr} 
Cartografia & da \\
implantação & $\mathrm{e}$ \\
execução & do \\
Programa & Saúde \\
na Escola & (PSE): \\
implicações para o \\
processo & de \\
\multicolumn{2}{l}{ desmedicalização. }
\end{tabular} & $\begin{array}{lr}\text { Educação } & \text { em } \\
\text { Revista } & \text { Belo } \\
\text { Horizonte. } & \end{array}$ & $\begin{array}{l}\text { Trata-se de um estudo } \\
\text { cartográfico. }\end{array}$ & $\begin{array}{lr}\text { Apresentar } & \text { uma } \\
\text { cartografia } & \text { da } \\
\text { implantação } & \text { e } \\
\text { execução } & \text { do } \\
\text { Programa Saúde na } \\
\text { Escola (PSE) e reus } \\
\text { efeitos para } \\
\text { processo } \\
\text { desmedicalização em } \\
\text { um município de } \\
\text { pequeno porte no sul } \\
\text { do Brasil. }\end{array}$ & $\begin{array}{l}\text { Por meio da articulação } \\
\text { intersetorial promovida } \\
\text { pelo Programa, } \\
\text { aproximaram-se os } \\
\text { setores Saúde, Educação e } \\
\text { Assistência Social. }\end{array}$ \\
\hline $\begin{array}{l}\text { Fontenele, Sousa, } \\
\text { Rasche, Souza, \& } \\
\text { Medeiros. (2017) }\end{array}$ & $\begin{array}{ll}\text { Construção } & \text { e } \\
\text { validação } & \\
\text { participativa } & \text { do } \\
\text { modelo lógico do } \\
\text { Programa Saúde } \\
\text { na Escola. }\end{array}$ & Saúde debate. & $\begin{array}{l}\text { Trata-se de um estudo } \\
\text { avaliativo, de abordagem } \\
\text { qualitativa. }\end{array}$ & $\begin{array}{l}\text { Construir e validar o } \\
\text { modelo lógico do } \\
\text { Programa Saúde na } \\
\text { Escola. }\end{array}$ & $\begin{array}{l}\text { A intersetorialidade ainda } \\
\text { se apresenta como o } \\
\text { desafio a ser vencido para } \\
\text { a execução do programa } \\
\text { nas áreas de abrangência. }\end{array}$ \\
\hline $\begin{array}{l}\text { Benedicto, Marim, } \\
\text { Simoes, \& Jorge. } \\
(2020)\end{array}$ & $\begin{array}{l}\text { Fatores de risco } \\
\text { para alterações na } \\
\text { comunicação e } \\
\text { queixas } \\
\text { comunicativas em } \\
\text { pré-escolares } \\
\text { durante ações do } \\
\text { Programa Saúde } \\
\text { na Escola. }\end{array}$ & Revista CEFAC. & $\begin{array}{l}\text { Estudo descritivo, } \\
\text { transversal, } \\
\text { retrospectivo, com } \\
\text { abordagem quantitativa. }\end{array}$ & 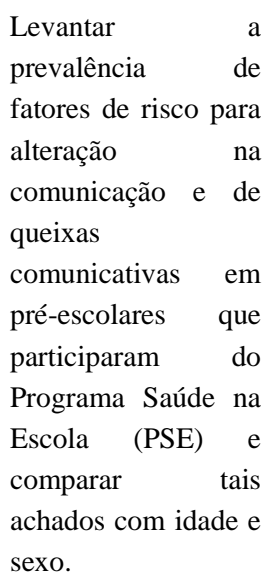 & $\begin{array}{l}\text { Em relação aos fatores de } \\
\text { risco investigados, o uso } \\
\text { de mamadeira foi o mais } \\
\text { citado, seguido das } \\
\text { infecções de ouvido ou } \\
\text { outros problemas no } \\
\text { ouvido. }\end{array}$ \\
\hline $\begin{array}{ll}\text { Vieira, } & \text { Dantas, } \\
\text { Miranda, } & \text { Araújo, } \\
\text { Monteiro, \& } & \text { Enders. } \\
(2018) & \end{array}$ & \begin{tabular}{l}
\multicolumn{2}{l}{ Intersetorialidade } \\
na promoção da \\
saúde escolar: um \\
estudo \\
Programa do \\
na
\end{tabular} & Saúde debate. & $\begin{array}{l}\text { Trata-se de pesquisa } \\
\text { qualitativa e exploratória. }\end{array}$ & $\begin{array}{l}\text { Analisar o Programa } \\
\text { Saúde na Escola no } \\
\text { distrito sanitário de } \\
\text { uma das capitais } \\
\text { brasileiras sob a ótica } \\
\text { da intersetorialidade }\end{array}$ & $\begin{array}{l}\text { Dentre os desafios, } \\
\text { destacam-se a necessidade } \\
\text { de processos de } \\
\text { monitoramento } \\
\text { avaliação do programa no } \\
\text { âmbito da promoção de }\end{array}$ \\
\hline
\end{tabular}




\begin{tabular}{|c|c|c|c|c|c|}
\hline & Escola. & & & $\begin{array}{l}\text { nas ações } \quad \text { de } \\
\text { promoção da saúde } \\
\text { escolar. }\end{array}$ & $\begin{array}{l}\text { saúde e a inserção ativa da } \\
\text { comunidade no campo. }\end{array}$ \\
\hline $\begin{array}{l}\text { Oliveira, Vargas, } \\
\text { Hartz, Dias, \& } \\
\text { Ferreira. (2018) }\end{array}$ & $\begin{array}{l}\text { Percepção de } \\
\text { escolares do } \\
\text { ensino } \\
\text { fundamental sobre } \\
\text { o Programa Saúde } \\
\text { na Escola: um } \\
\text { estudo de caso em } \\
\text { Belo Horizonte, } \\
\text { Brasil. }\end{array}$ & $\begin{array}{l}\text { Ciência \& Saúde } \\
\text { Coletiva. }\end{array}$ & Estudo qualitativo. & $\begin{array}{l}\text { Investigar } r \text { a } \\
\text { percepção que os } \\
\text { escolares brasileiros } \\
\text { possuem em relação } \\
\text { às atividades } \\
\text { desenvolvidas pelo } \\
\text { Programa Saúde na } \\
\text { Escola. }\end{array}$ & $\begin{array}{l}\text { Os escolares se mostraram } \\
\text { passivos e receptivos sem, } \\
\text { no entanto, } \\
\text { protagonismo necessário à } \\
\text { produção da própria } \\
\text { saúde. }\end{array}$ \\
\hline $\begin{array}{l}\text { Sousa, Esperdião, \& } \\
\text { Medina. (2017) }\end{array}$ & $\begin{array}{l}\text { A } \\
\text { intersetorialidade } \\
\text { no Programa } \\
\text { Saúde na Escola: } \\
\text { avaliação ro do } \\
\text { processo político- } \\
\text { gerencial e das } \\
\text { práticas } \\
\text { trabalho. }\end{array}$ & $\begin{array}{l}\text { Ciência \& Saúde } \\
\text { Coletiva. }\end{array}$ & Estudo avaliativo. & \begin{tabular}{lr} 
Analisar a & ação \\
intersetorial & \\
desenvolvida & entre \\
os setores saúde & e \\
educação & no \\
processo & de \\
implementação & do \\
Programa Saúde & na \\
Escola & em \\
municípios da região \\
metropolitana do \\
\multicolumn{2}{l}{ Nordeste brasileiro. }
\end{tabular} & $\begin{array}{l}\text { O programa fortaleceu a } \\
\text { relação entre os dois } \\
\text { setores, entretanto, } \\
\text { aspectos da articulação } \\
\text { intersetorial no processo } \\
\text { político-gerencial e nas } \\
\text { práticas mostraram } \\
\text { fragilidades e limitações. }\end{array}$ \\
\hline $\begin{array}{l}\text { Chiari, } \\
\text { Akerman, } \\
\begin{array}{l}\text { Machado } \\
\text { (2018) }\end{array}\end{array}$ & $\begin{array}{l}\text { Rede intersetorial } \\
\text { do Programa } \\
\text { Saúde na } \\
\text { Escola: sujeitos, } \\
\text { percepções e } \\
\text { práticas. }\end{array}$ & Cad. Saúde Pública. & $\begin{array}{l}\text { Foi desenvolvido um } \\
\text { estudo de caso por meio } \\
\text { de pesquisa documental e } \\
\text { da aplicação de um } \\
\text { questionário eletrônico. }\end{array}$ & 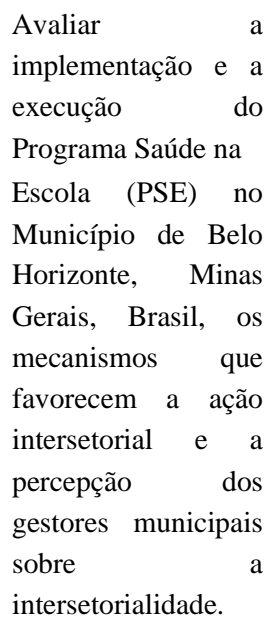 & $\begin{array}{l}\text { Percebeu-se uma } \\
\text { dificuldade na construção } \\
\text { da intersetorialidade no } \\
\text { desenvolvimento das } \\
\text { ações do PSE. }\end{array}$ \\
\hline $\begin{array}{l}\text { Santos, Gasparim, } \\
\text { Monteiro, Brito, \& } \\
\text { Silva. (2019) }\end{array}$ & $\begin{array}{l}\text { Relato de } \\
\text { Experiência: } \\
\text { Construção e } \\
\text { Desenvolvimento } \\
\text { do Programa de } \\
\text { Saúde na } \\
\text { Escola (PSE) sob } \\
\text { a Perspectiva da } \\
\text { Sexualidade na } \\
\text { Adolescência. }\end{array}$ & $\begin{array}{l}\text { Revista brasileira de } \\
\text { educação médica. }\end{array}$ & $\begin{array}{l}\text { Relato de experiência de } \\
\text { abordagem crítico- } \\
\text { reflexiva. }\end{array}$ & 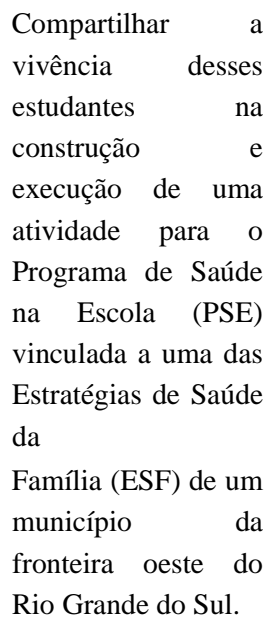 & $\begin{array}{l}\text { São importantes ações de } \\
\text { promoção de saúde, uma } \\
\text { vez que visualiza-se o } \\
\text { amplo alcance de ações } \\
\text { como as desenvolvidas } \\
\text { por meio desses } \\
\text { programas e o impacto } \\
\text { que podem trazer para a } \\
\text { saúde da comunidade. }\end{array}$ \\
\hline
\end{tabular}




\begin{tabular}{|c|c|c|c|c|c|}
\hline $\begin{array}{l}\text { Alves, Araújo, Costa, } \\
\text { Marques, \& Alencar. } \\
\text { (2019) }\end{array}$ & $\begin{array}{l}\text { Ação } \\
\text { interdisciplinar de } \\
\text { promoção à saúde } \\
\text { no programa } \\
\text { escola da família: } \\
\text { relato } \\
\text { experiência de de } \\
\text { residentes do } \\
\text { programa } \\
\text { multidisciplinar } \\
\text { em saúde da } \\
\text { família. }\end{array}$ & Revista Nursing. & Relato de experiência. & $\begin{array}{l}\text { Relatar a experiência } \\
\text { vivida por residentes } \\
\text { multiprofissionais } \\
\text { em saúde da família a } \\
\text { partir da participação } \\
\text { no Programa Escola } \\
\text { da Família. }\end{array}$ & $\begin{array}{l}\text { A prática interdisciplinar é } \\
\text { necessária para enriquecer } \\
\text { as atividades propostas. }\end{array}$ \\
\hline $\begin{array}{l}\text { Fernandes \& Köptcke. } \\
\text { (2021) }\end{array}$ & $\begin{array}{l}\text { Análise da ação de } \\
\text { saúde ocular do } \\
\text { Programa Saúde } \\
\text { na } \\
\begin{array}{ll}\text { Escola no } & \text { Brasil } \\
\text { de } 2014 \text { a } & 2019: \\
\text { um } & \text { estudo } \\
\text { transversal. }\end{array}\end{array}$ & $\begin{array}{l}\text { Epidemiol. Serv. } \\
\text { Saude, }\end{array}$ & $\begin{array}{ll}\text { Estudo } & \text { transversal } \\
\text { descritivo. } & \end{array}$ & 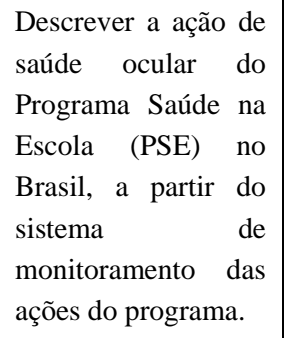 & $\begin{array}{l}\text { As intervenções de saúde } \\
\text { ocular do PSE têm } \\
\text { crescido ao longo dos } \\
\text { anos no país, contribuindo } \\
\text { para a prevenção da } \\
\text { deficiência visual e } \\
\text { cegueira dos estudantes } \\
\text { brasileiros. }\end{array}$ \\
\hline Fontenele. (2017) & $\begin{array}{l}\text { Avaliação } \\
\text { colaborativa das } \\
\text { ações de saúde do } \\
\text { Programa Saúde } \\
\text { na Escola carioca. }\end{array}$ & $\begin{array}{lr}\text { Escola } & \text { de } \\
\text { Enfermagem Anna } \\
\text { Nery. }\end{array}$ & $\begin{array}{l}\text { Pesquisa avaliativa com } \\
\text { abordagem qualitativa. }\end{array}$ & $\begin{array}{l}\text { Avaliar, de forma } \\
\text { colaborativa com os } \\
\text { gestores } \\
\text { executores, as }\end{array}$ & $\begin{array}{l}\text { As intervenções } \\
\text { favoreceram } \\
\text { entendimento dos } \\
\text { objetivos do programa e } \\
\text { contribuíram na discussão } \\
\text { sobre a produção, os } \\
\text { resultados e impactos } \\
\text { esperados na execução, } \\
\text { enaltecendo o Programa } \\
\text { Saúde na Escola e a } \\
\text { Atenção Básica }\end{array}$ \\
\hline $\begin{array}{l}\text { Pereira, Pereira, Faria, } \\
\text { Cordeiro, Lanza, \& } \\
\text { Viegas. (2020) }\end{array}$ & 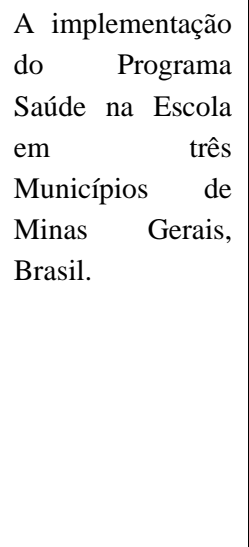 & $\begin{array}{l}\text { Revista de } \\
\text { Enfermagem do } \\
\text { Centro - Oeste } \\
\text { Mineiro. }\end{array}$ & $\begin{array}{l}\text { Estudo de } \begin{array}{r}\text { casos } \\
\text { múltiplos } \\
\text { qualitativos. }\end{array} \\
\text { holístico- }\end{array}$ & 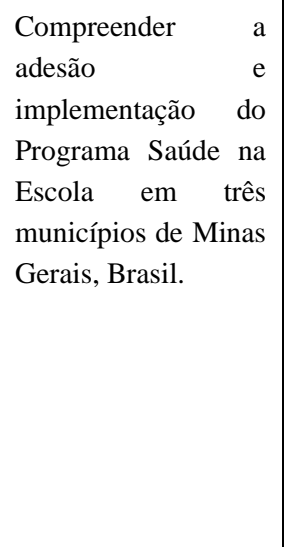 & $\begin{array}{l}\text { Vincular ações de saúde } \\
\text { ao cotidiano dos escolares } \\
\text { é uma tarefa intensa. Ao } \\
\text { analisar o contexto do } \\
\text { PSE e o cotidiano de } \\
\text { trabalho expresso pelos } \\
\text { profissionais da Saúde e } \\
\text { da } \\
\text { Educação, observaram-se } \\
\text { diferentes impasses e } \\
\text { formas de implementação } \\
\text { prática frente à sua } \\
\text { gênese. }\end{array}$ \\
\hline
\end{tabular}




\begin{tabular}{|c|c|c|c|c|c|}
\hline $\begin{array}{l}\text { Medeiros Pinto, Paiva, } \\
\text { Nascimento, } \\
\text { Rebouças, \& Silva, } \\
(2018)\end{array}$ & $\begin{array}{lr}\text { Facilidades } & \text { e } \\
\text { dificuldades } & \text { na } \\
\text { implantação } & \text { do } \\
\text { Programa } & \text { Saúde } \\
\text { na Escola em } & \text { um } \\
\text { município } & \text { do } \\
\text { nordeste } & \text { do } \\
\text { Brasil. } & \end{array}$ & Rev Cuid. & $\begin{array}{l}\text { Pesquisa descritiva, } \\
\text { transversal, quantitativa. }\end{array}$ & $\begin{array}{lr}\text { Identificar } & \text { as } \\
\text { facilidades } & \text { e } \\
\text { dificuldades } & \text { na } \\
\text { implantação } & \text { do } \\
\text { Programa Saúde } & \text { na } \\
\text { Escola em um } \\
\text { município } \\
\text { nordeste brasileiro. }\end{array}$ & $\begin{array}{lrr}\text { A satisfação } & \text { dos } \\
\text { profissionais } & \text { e } & \text { a } \\
\text { articulação entre } & \text { os } \\
\text { setores saúde } & \text { educação } \\
\text { são } & \text { elementos } \\
\text { facilitadores } & \text { na } \\
\text { implantação do Programa } \\
\text { Saúde na Escola. }\end{array}$ \\
\hline $\begin{array}{ll}\text { Vieira, } & \text { Dantas, } \\
\text { Miranda, } & \text { Araújo, } \\
\text { Monteiro, \& } & \text { Enders. } \\
(2018) & \end{array}$ & $\begin{array}{lr}\text { Programa de } \\
\text { Enfermagem } \\
\text { Saúde na Escola: } \\
\text { prevenção r e } \\
\text { controle de } \\
\text { sobrepeso/obesida } \\
\text { de } \\
\text { adolescentes. }\end{array}$ & $\begin{array}{ll}\text { Rev Esc Enferm } \\
\text { USP. }\end{array}$ & $\begin{array}{l}\text { Estudo metodológico } \\
\text { qualitativo. }\end{array}$ & \begin{tabular}{l}
\multicolumn{2}{l}{ Descrever o processo } \\
de construção de \\
uma intervenção na \\
forma de um \\
Programa \\
Atenção de \\
Enfermagem para \\
Adolescentes \\
voltado à prevenção \\
e controle do excesso \\
de peso/obesidade no \\
ambiente escolar.
\end{tabular} & $\begin{array}{lr}\text { A intervenção } & \text { de } \\
\text { enfermagem } & \text { orientada } \\
\text { pelo Mapeamento de } & \text { dntervenção permite ações } \\
\text { embasadas em } & \text { teorias, } \\
\text { participação } & \text { da } \\
\text { comunidade, } & \text { vínculo } \\
\text { escola/serviço } & \mathrm{e} \\
\text { continuidade } & \mathrm{da} \\
\text { assistência. } & \end{array}$ \\
\hline
\end{tabular}

Fonte: Araújo, Nogueira, Santos, Monteiro, Vieira, Rodrigues, Quadros, Freitas, Oliveira \& B et al (2021).

\section{Discussão}

Conforme a Organização Mundial de Saúde a possibilidade de abertura do espaço escolar aos objetivos da saúde como prioridade de caráter público baseia-se no entendimento de que um programa de saúde estudantil efetivo se apresenta como uma aposta mais promissora do país para que se alcance melhores resultados tanto no campo da educação como no da saúde. Dessa maneira, numa perspectiva global, os desafios indicados como os mais importantes na etiologia de morbimortalidade entre crianças e também em adultos, podem assim, ser prevenidos ou mesmo minimizados com o auxílio de programas de saúde escolar e saúde dos jovens (WHO, 2017).

O PSE propõe uma articulação e integração entre saúde e educação por meio de ações que contribuam com o fortalecimento da promoção a saúde no âmbito escolar, de forma a melhorar a qualidade de vida de crianças, adolescentes e jovens por meio de programas e projetos que possam promover e contribuir no desenvolvimento destes cidadãos considerando os diversos contextos: políticos, econômicos, sociais e culturais da população brasileira (Brasil, 2015).

Compreende-se a escola com um espaço privilegiado de troca de saberes, reflexão e formulação de uma consciência crítica, sendo ela favorável às ações de educação em saúde (Silva, Almeida, Machado, Silva, Cardoso, Costa, \& Cotta, 2019). No país, os ideais da saúde na escola foram inseridos nos projetos brasileiros conforme as políticas públicas foram sendo consideradas junto a iniciativa de caráter global, conhecido como escolas promotoras de saúde (Silva, Almeida, Machado, Silva, Cardoso, Costa, \& Cotta, 2019).

Nessa discussão, a escola se apresenta com um espaço cada vez mais associado a objetivos políticos públicos de caráter intersetorial propícios à promoção à saúde. Assim, vale, dessa forma, intensificar o entendimento acerca do contexto, identificar as ações produzidas, bem como compreender seus limites e possibilidades no desenvolvimento das experiências vividas (Vieira \& Belisário, 2018).

As políticas de saúde no âmbito brasileiro têm acontecido na afirmação da intersetorialidade. Outrora, em outro cenário histórico, discutiu-se tanto sobre saúde e promoção da saúde como no contexto atual, o que reafirma a valorização do papel de promoção da saúde associada à escola como agente de transformação da realidade. Dessa forma, corrobora-se a exigência de 
atuação não apenas do setor saúde, mas também como o resultado de intervenções intersetoriais e de caráter multidisciplinar, tornando a intersetorialidade como uma peça indispensável para a prática da promoção da saúde (Ventura, Alencar, Araújo, \& Pinheiro, 2019).

O PSE apresenta-se oficialmente como uma política de aspecto intersetorial, o que determina que a responsabilidade por sua ação deve ser dividida entre os setores de educação e saúde, sobretudo, entre profissionais que atuam nas escolas e nas Unidades Básicas de Saúde. Conforme suas diretrizes, a força da intersetorialidade apoia-se na possibilidade de associação entre os dispositivos públicos da educação e da saúde (Silveira, Estermann \& Félix, 2019).

Este programa é uma proposta global, que vale-se da ideia de que a comunidade na qual está inserida e a sociedade na qual se relaciona, idealmente, a saúde na escola não se vale de intervenções desconexas e assistemáticas, mas associa provisão de recursos específicos, intervenções periódicas e estratégias intersetoriais (Silva, \& Bodstein, 2016).

Políticas e programas públicos voltados para a área da saúde e educação são essenciais para a construção da consciência cidadã e para melhores índices de qualidade de vida e saúde da população. Índices mais satisfatórios de educação estão associados a uma população mais saudável, bem como, uma população com melhores indicadores de saúde tem maiores chances de se apropriar de conhecimentos e saberes da educação informal e formal (Casemiro, Fonseca \& Secco, 2014).

A escola, além de possibilitar a transmissão de saberes sobre a saúde, divididas em disciplinas, deve ainda, ensinar e desenvolver valores e atitudes críticas em relação à realidade social e aos estilos de vida, em ambiente de produção de habilidades que balizem as aprendizagens no decorrer da vida e que, promovam a autonomia e o empoderamento para a promoção da saúde (Brasil, 2015).

A intersetorialidade vem sendo destacada, há certo tempo, como uma ferramenta para organizar recursos de inúmeras ordens em relação ao planejamento, implementação e monitoramento de políticas públicas. Permite analisar e resolver desafios que apresentam muitas causas, diretamente porque se relaciona com formas de planejar, implementar e monitorar políticas públicas que determinam saídas conjuntas e articuladas para desafios que afetam duas ou mais áreas (Ventura, Alencar, Araújo, \& Pinheiro, 2019).

Nessa interface, a Atenção Básica na figura da Estratégia Saúde da Família deve exercer as ações em uma ou mais escolas de seu território de abrangência e essa atuação deve ser alvo de acordo e planejamento com as instituições escolares. No documento denominado Agenda, Educação e Saúde, construído em forma ilustrada e em tipo passo a passo, sugere-se, por exemplo, que ocorra a circulação conjunta de profissionais das escolas e das equipes de saúde da família aos redores das escolas para avaliação e aproximação com a realidade local (Silva, 2019).

Para essa tarefa, os profissionais devem estar portando materiais como câmeras fotográficas, blocos de anotações e mapas e fazer a anotação dos desafios e barreiras visualizados nas ruas, tais como, lixões a céu aberto, ausência de asfalto e saneamento básico, dentre outros, com o objetivo de esses empasses sejam discutidos de forma conjunta entre as equipes de saúde da família e os membros das equipe escolar (Silva, 2019).

O documento indica ainda que, com essas anotações, os profissionais elaborem relatórios acerca da situação das regiões visitadas e, então, dialoguem entre si e com a comunidade escolar como forma de resolução dos desafios encontrados. Desse diálogo, surgiria o projeto que validaria a atuação conjunta das equipes, de maneira a conectar e associar as diferentes estratégias do PSE com a "realidade" para, assim, transformá-la, promovendo a saúde dos alunos das escolas públicas (Silva, 2019).

Assim, essa articulação entre os membros da escola e a os profissionais que compõem a equipe de saúde da família é avaliado como uma ferramenta essencial para envolver a comunidade local e construir, entre os alunos e seus familiares, habilidade individuais (e familiares) para lidar com a própria saúde, dentro da perspectiva do que se define, no âmbito do PSE, de qualidade de vida (Silveira, Estermann, \& Félix, 2019).

Nesse contexto, indica-se que a escola tenha gestão e divisão da responsabilidade na promoção da saúde dos estudantes 
que dela fazem parte, compreendendo-se que a escola não é espaço de assistência em saúde como é o caso da unidade básica de saúde. De acordo com as premissas do programa, as duas áreas associadas necessitam ocupar-se de determinada vertente em relação à promoção da saúde na comunidade escolar (Silveira, Estermann, \& Félix, 2019).

Compreende-se que, com a ação de ensinar por meio de "sua realidade", o aluno seja captado para enxergar sentido no que lhe é oferecido e que, ao envolver-se, aprenda, nesse circunstância, a cuidar-se. Ressalta-se que a perspectiva "por meio da realidade do aluno", no seio do PSE, assume espaço e posiciona profissionais da saúde e educação para indivíduos que necessitam se envolver para realizar uma leitura da realidade do território em que se encontram a Unidade Básica de Saúde e as escolas (Silveira, Estermann, \& Félix, 2019).

Assim, o PSE apresenta-se como uma nova proposta de complemento de uma exigência há tempos debatida: a intensificação no processo de integração entre os campos da saúde e educação, possibilitando a intersetorialidade discutida pelo Sistema Único de Saúde (SUS) e a responsabilidade compartilhada entre essas áreas, que frequentemente atuam de maneira isolada (Brasil, 2017).

Dessa forma, realizar-se a união da intersetorialidade - sabidamente conhecida como uma das diretrizes da Política Nacional de Promoção da Saúde e ratificada pela Política Nacional de Atenção Básica (PNAB) como fundamental para o processo de construção das ações em saúde - ao fértil e rico ambiente escolar é um plano benefício de construção de saúde (Brasil, 2017).

Para que as ações possam ser desenvolvidas a parceria entre os profissionais da saúde e educação é essencial, para que assim se possa planejar as ações do PSE e efetivá-las dentro do ambiente escolar promovendo o conhecimento em saúde por meio da promoção e prevenção da saúde (Brasil, Silva, \& Rodrigues, 2017). Os gestores públicos podem ser considerados como importantes atores para o processo de coordenação de intervenções de caráter intersetorial, uma vez que, em uma escala mais local há possibilidade de realizar a união de personagens de importante inserção social em torno de desafios comuns aquela realidade e obter respostas eficientes, dirigindo o esforço conjunto para intervenções que acarretem melhoria na qualidade de vida da população (Farias, Sá, Figueiredo, \& Filho 2016).

Nessa discussão, são apontados como fatores que assumem papel de destaque para a implantação efetiva do PSE, dentre outros, a realização de atividades de forma conjunta entre os profissionais do campo da educação e da saúde, a satisfação em realizar essas atividades, bem como a chance de possibilitar a continuidade da assistência aos alunos. A participação e contribuição da equipe multiprofissional e a disposição de aporte financeiro e material também são fatores que contribuem para a implementação do PSE conforme desejados pelos atores envolvidos (Medeiros, Pinto, Paiva, Nascimento, Rebouças, \& Silva, 2018).

Por fim, fatores como o tempo, a falta de conhecimento sobre o programa, recursos e planejamento podem afetar o desenvolvimento e implantação das ações do PSE. Alcançar a integralidade e estimular o saber em saúde das crianças, adolescentes e jovens frequentadores do espaço escolar só é possível a partir de uma colaboração entre os setores da saúde e educação na busca de objetivos comuns a fim de alcançar melhores resultados na promoção, prevenção e assistência destes escolares sendo estes o propósito do PSE, que quando bem articulado é capaz de desenvolver ações com melhor resultado no desenvolvimento da qualidade e condições de vida desses escolares (Souza, Esperidião, \& Medina, 2017).

Nesse contexto, a criação de processos de comunicação entre as áreas é tarefa essencial. A agenda institucional necessita ser readequada para que a educação e a saúde possam, de forma inequívoca, aproximar-se. Além disso, a agenda entre esses setores necessita assumir o papel em relação à relevância política necessária (Chiari, Ferreira, Akerman, Amaral, Machado \& Senna, 2018). 


\section{Conclusão}

Ainda prevalece a ausência de comunicação entre os setores saúde e educação, uma vez que cada um tem suas próprias metas a serem alcançadas, dificultando o processo de integração dos profissionais para o desenvolvimento do Programa Saúde na Escola. Frequentemente, as ações desenvolvidas nas escolas são pontuais e assistemáticas, apenas como respostas a demandas específicas, o que limita o alcance das ações do Programa Saúde na Escola, fazendo que a intersetorialidade não ocorra de fato. Nesse sentido, sugere-se a realização de novos estudos exploratórios que investiguem as práticas em relação ao programa para possibilitar avanços na assistência em saúde no ambiente escolar.

\section{Referências}

Alves, M. L., Araújo, H. P. A., Costa, L. A. S., Marques, M. C. S., \& Alencar, R. A. (2019). Ação interdisciplinar de promoção à saúde no programa escola da família: relato de experiência de residentes do programa multidisciplinar em saúde da família: Revista Nursing, 22(252), 2875-2877.

Batista, A. S. M., Mondini, 1., \& Jaime, C. P. (2017). Ações do Programa Saúde na Escola e da alimentação escolar na prevenção do excesso de peso infantil: experiência no município de Itapevi, São Paulo, Brasil, 2014: Epidemiol, 26(3), 569-578.

Barbieri, F. A., \& Noma, K. A. (2017). A função social do Programa Saúde na Escola: formação para a nova sociabilidade do capital? Perspectiva, 35 (1), 161 187.

Brasil. (2017). Ministério da Saúde. Secretaria de Atenção à Saúde. Departamento de Atenção Básica. Instrutivo PSE. Ministério da Saúde. Secretaria de Atenção à Saúde. Departamento de Atenção Básica. Ministério da Saúde.

Brasil. (2009). Ministério da Saúde. Secretaria de Atenção à Saúde. Departamento de atenção básica. Cadernos de Atenção Básica. Saúde na Escola. Brasília: Ministério da Saúde.

Brasil. (2021). Ministério da Saúde. Secretaria de Vigilância em Saúde. Departamento de Análise de Situação de Saúde. Plano de ações estratégicas para o enfrentamento das doenças crônicas não transmissíveis (DCNT) no Brasil 2011-2022. Brasília: Ministério da Saúde, 2021.

Brasil. (2015). Ministério da Saúde. Caderno do gestor do PSE. Ministério da Saúde, Ministério da Educação. Ministério da Saúde.

Silva, C. S. (2019). Saúde na escola: intersetorialidade e promoção da saúde. Editora Fiocruz.

Brambilla, K. D., Kleb, E. M., \& Magro, D. P. L. M. (2020). Cartografia da implantação e execução do Programa Saúde na Escola (PSE): implicações para o processo de desmedicalização: Educação em Revista Belo Horizonte, 1(36), 3-14.

Benedicto, M. N., Marim, C. G., Simoes, O. H., \& Jorge, M. T. (2020). Fatores de risco para alterações na comunicação e queixas comunicativas em préescolares durante ações do Programa Saúde na Escola: Revista CEFAC, 22(6), 1-8.

Casemiro, J. P., Fonseca, C. B, A., \& Secco, M. V. F. (2014). Promover saúde na escola: reflexões a partir de uma revisão sobre saúde escolar na América Latina: Saúde Colet, 19(3), 829-840.

Chiari, G. P. A. Ferreira, C. R., Akerman, M., Amaral, L. H. J., Machado. M. K. \& Senna, B. I. M. (2018). Rede intersetorial do Programa Saúde na Escola: sujeitos, percepções e práticas: Cad. Saúde Pública, 34(5), 1-15.

Farias, V. C. I., Sá, F. P. M. R., Figueiredo, N. \& Filho, M. A. (2016). Análise da Intersetorialidade no Programa Saúde na Escola. Revista Brasileira de Educação Médica, 40(2), 261-267.

Fernandes, A. L., \& Köptcke, L. S. (2019). Análise da ação de saúde ocular do Programa Saúde na Escola no Brasil de 2014 a 2019: um estudo transversal: Epidemiol. Serv. Saúde, 2130(2), 1-11.

Fontenele, R. M., Sousa, I. A., Rasche, S. A. Souza, N. H. M., \& Medeiros, C. D. (2017). Construção e validação participativa do modelo lógico do Programa Saúde na Escola: Saúde Debate, 41(1), 167-179.

Fontenele, R. M. (2017). Avaliação colaborativa das ações de saúde do Programa Saúde na Escola Carioca. RJ. Tese (Doutorado em Enfermagem): Escola de Enfermagem Anna Nery, Universidade Federal do Rio de Janeiro, 195 p.

Instituto Brasileiro de Geografia e Estatística. (2016). Pesquisa nacional de saúde do escolar. Pesquisa nacional de saúde do escolar 2015. Rio de Janeiro: IBGE.

Brasil, E. G. M., Silva, M. R., Silva, F. R. M., \& Rodrigues, P. D. (2017). Promoção da saúde de adolescentes e Programa Saúde na Escola: complexidade na articulação saúde e educação: Rev Esc Enferm USP, 51 (1), 1-9.

Lopes, E. I., Nogueira, D. A.J., \& Rocha, G. D. (2018). Eixos de ação do Programa Saúde na Escola e Promoção da Saúde: revisão integrativa: Saúde Debate Rio de Janeiro, 42(118), 773-789.

Medeiros, E. R., Pinto, G. S. E., Paiva. S. C. A., Nascimento, A. P. C., Rebouças, C. G.D., \& Silva, B. Y. S. (2018). Facilidades e dificuldades na implantação do Programa Saúde na Escola em um município do nordeste do Brasi: Rev Cuid, 9(2), 2127-2134. 
Silva, M.R. I., Almeida, P. A., Machado, C. J., Silva, S. L., Cardoso, F. A. J., Costa, D. G., \& Cotta, M. M. R. (2019). Processo de Acreditação das Escolas Promotoras de Saúde em âmbito mundial: revisão sistemática: Ciênc. saúde colet, 24 (2), 475-486.

Oliveira, L. S. P. F., Vargas, D. M. A., Hartz, Z., Dias, S., \& Ferreira, F. E. (2018). Percepção de escolares do ensino fundamental sobre o Programa Saúde na Escola: um estudo de caso em Belo Horizonte, Brasil: Ciência \& Saúde Coletiva, 23(9), 2891-2898.

Pereira, P. L. G., Pereira, D. M., Faria, S. G. R., Cordeiro, R. D., Lanza, M. F., \& Viegas, F. M. S. (2020). A implementação do programa saúde na escola em três municípios de Minas Gerais, Brasil. Revista de Enfermagem do Centro Oeste Mineiro, 10 (10), 1-11.

Silva, A. L., Reis, C. E., \& Milhorine, R. B. (2016). Atuação do Enfermeiro na Promoção da Saúde Escolar. II Congresso Interdisciplinar de Promoção da Saúde. Universidade de Santa Cruz do Sul (UNISC).

Silveira, C. C., Estermann, E. D., \& Félix, J. M. (2019). A generificação da intersetorialidade no Programa Saúde na Escola. Rev. bras. Estud. pedagog, 100(255), 423-442.

Santos, D. C. A., Gasparim, A., Monteiro, M. G., Brito, M. R., \& Silva, M. A. V. (2019). Construção e Desenvolvimento do Programa de Saúde na Escola (PSE) sob a Perspectiva da Sexualidade na Adolescência Relato de Experiência: Rev. bras. educ. med, 43 4),

Silva, C. S. \& Bodstein, R. C. A. (2019). A theoretical framework on intersectoral practice in school health promotion: Ciênc. Saúde Colet, 21(6), 1777-1788.

Ventura, A. N. G. F., Alencar, M. R., Araújo, S. I., \& Pinheiro, R. W. (2019). A Estratégia de Saúde da Família e o diálogo sobre a Intersetorialidade: Id on Line Rev. Mult. Psic, 13(47): 63-76.

Sousa, M. C., Esperdião, A. M., \& Medina, G. M. (2017). A intersetorialidade no Programa Saúde na Escola: avaliação do processo político-gerencial e das práticas de trabalho: Ciência \& Saúde Coletiva, 22(6), 1781-1790.

Vieira, S. L., \& Belisário, A. S. (2018). Intersetorialidade na promoção da saúde escolar: um estudo do Programa Saúde na Escola Saúde debate: Saúde Debate, 42(4), 120-133.

Vieira, C. E. N. K., Dantas, A. N., Miranda, V. M. S. L., Araújo, C. K. A., Monteiro, I. A., \& Enders, C. B. (2018). School Health Nursing Program: prevention and control of overweight/obesity in adolescents: Rev Esc Enferm USP, 52(5), 1-8.

Wang, G., Zhang, Y., Zhao, J., Zhang, J., \& Jiang, F. (2020). Mitigate the effects of home confinement on children during the COVID-19 outbreak: Lancet, 395(10228), 945-947.

World Health Organization. (2017. Growing up unequal: Gender and socioeconomic differences in young people's health and well-being. Health policy for children and adolescents. Health Behaviour in School-aged Children (HBSC) study: international report from the 2013/2014 survey. 